\title{
Miniaturization of Inductively Coupled Plasma Sources
}

\author{
Yu Yin, Jason Messier, and Jeffrey A. Hopwood, Member, IEEE
}

\begin{abstract}
The scaling laws associated with the miniaturization of planar inductively coupled plasmas (ICP's) are investigated. The applications for miniature ICP's include microelectromechanical systems (MEMS) for chemical analysis and micro ion propulsion systems. Langmuir probe and microwave interferometry measurements of three ICP's with spiral-shaped coil diameters of 5,10 , and $15 \mathrm{~mm}$ show that electron density typically falls in the range of $10^{16}-10^{17} \mathrm{~m}^{-3}$. The electron density is about an order of magnitude lower than large-scale ICP's as a result of the large surface-to-volume ratio of small discharges. The measured electron temperature is higher than predicted by a simple "global model" unless the plasma dimensions are determined more precisely by subtracting the sheath width from the chamber dimensions. Since the sheath width does not scale with the plasma size, the sheath width may ultimately limit the minimum size of ICP's. Plasma initiation power is determined to have a minimum at a gas pressure for which the electron collision frequency equals the frequency of the RF power supply. Small scale ICP's operating at $460 \mathrm{MHz}$ can therefore be started most easily at $\sim 1$ torr. The design of the coil is critical to miniature ICP performance. Unlike large-scale ICP's that operate efficiently using a broad range of coil shapes, the miniature coil must be carefully designed and constructed to minimize parasitic resistance.
\end{abstract}

Index Terms - Inductively coupled plasma (ICP), microelectromechanical systems (MEMS).

\section{INTRODUCTION}

$\mathbf{R}$ ECENTLY, the demand for plasma processing equipment capable of uniformly treating large wafers has driven the development of larger plasma sources. The latest goal is the uniform plasma treatment of $300 \mathrm{~mm}$ diameter wafers which requires a plasma source diameter considerably larger than $300 \mathrm{~mm}$. In this paper, however, the opposite direction is investigated. We will describe the scaling-down of a plasma source to dimensions that are ultimately compatible with its use in microelectromechanical systems (MEMS).

The desirable characteristics of a miniaturized MEMS plasma are similar to its large-scale counterparts. For example, the source should have the ability to operate using a reactive gas for long periods of time. This precludes small plasma sources that depend on electrodes or specially treated surfaces such as ac plasma displays [1]. In addition, the power dissipation should be minimal and the operating voltage should preferably be low and compatible with complementary

Manuscript received January 26, 1999; revised May 14, 1999. This work was supported by the National Science Foundation under Grant ECS-9701916.

The authors are with the Department of Electrical and Computer Engineering, Northeastern University, Boston, MA 02115 USA (e-mail: hopwood@ece.neu.edu).

Publisher Item Identifier S 0093-3813(99)08723-8. metal-oxide-semiconductor (CMOS) circuits. An inductively coupled plasma is a reasonable choice since it is electrodeless and, with a characteristic input impedance of $50 \Omega$ (as seen by the RF generator), requires only $\pm 10 \mathrm{~V}$ dc power supplies to deliver $1 \mathrm{~W}$ of $\mathrm{RF}$ power to the plasma.

Applications of miniaturized plasma sources include analytical instrumentation such as emission and mass spectroscopy. For example, Ross et al. [2] have characterized a 9-mm inductively coupled microtorch for mass spectrometry. As analytical instruments are reduced in size and fabricated using MEMS technology, the need for MEMS-compatible sources of gas excitation and ionization is obvious. Another potential application of miniaturized plasma sources is the production of small ion beams used for the generation of thrust [3]. For the analytical applications, it may be useful to operate the plasma at relatively high pressures (10 s of torr), since miniaturized pumping systems will likely be inefficient at creating high vacuum environments. On the other hand, ion thruster applications will need plasma sources that operate at low pressure $\left(\sim 10^{-3}\right.$ torr) to insure efficient mass utilization. One of the goals of this work is to determine the scaling of operating pressure and plasma size for miniature inductively coupled plasmas.

In this paper three scaled-down ICP's are investigated with pancake-shaped coils of 5, 10 and $15 \mathrm{~mm}$ diameter. Largescale ICP's typically operate by electrically resonating the coil at a frequency of $13.56 \mathrm{MHz}$. The optimum frequency for plasma generation is found to increase to $460 \mathrm{MHz}$ as the coil diameter is reduced to $5 \mathrm{~mm}$. The electron density produced by small ICP's is dependent on frequency, but the electron temperature and plasma potential are independent of frequency. The variation of electron density is attributed to the frequency-dependent quality factor of the coil, $Q(f)$. The electron temperature is measured and found to compare favorably with a global model when the plasma dimensions are determined by subtracting the sheath width from the chamber dimensions. Since the sheath width does not scale, the limits of dimensional scaling will be determined by the plasma sheath.

\section{EXPERIMENT}

\section{A. Exploratory Scaling}

The initial attempt at a miniature ICP was formed from a 20 -turn coil wound around a $6 \mathrm{~mm}$ Pyrex tube. The plasma was confined within the $4 \mathrm{~mm}$ i.d. of the tube. Two tungsten probes pierced the tube, allowing for the determination of electron temperature $\left(T_{e}\right)$ and ion density $\left(n_{i}\right)$ from the double 


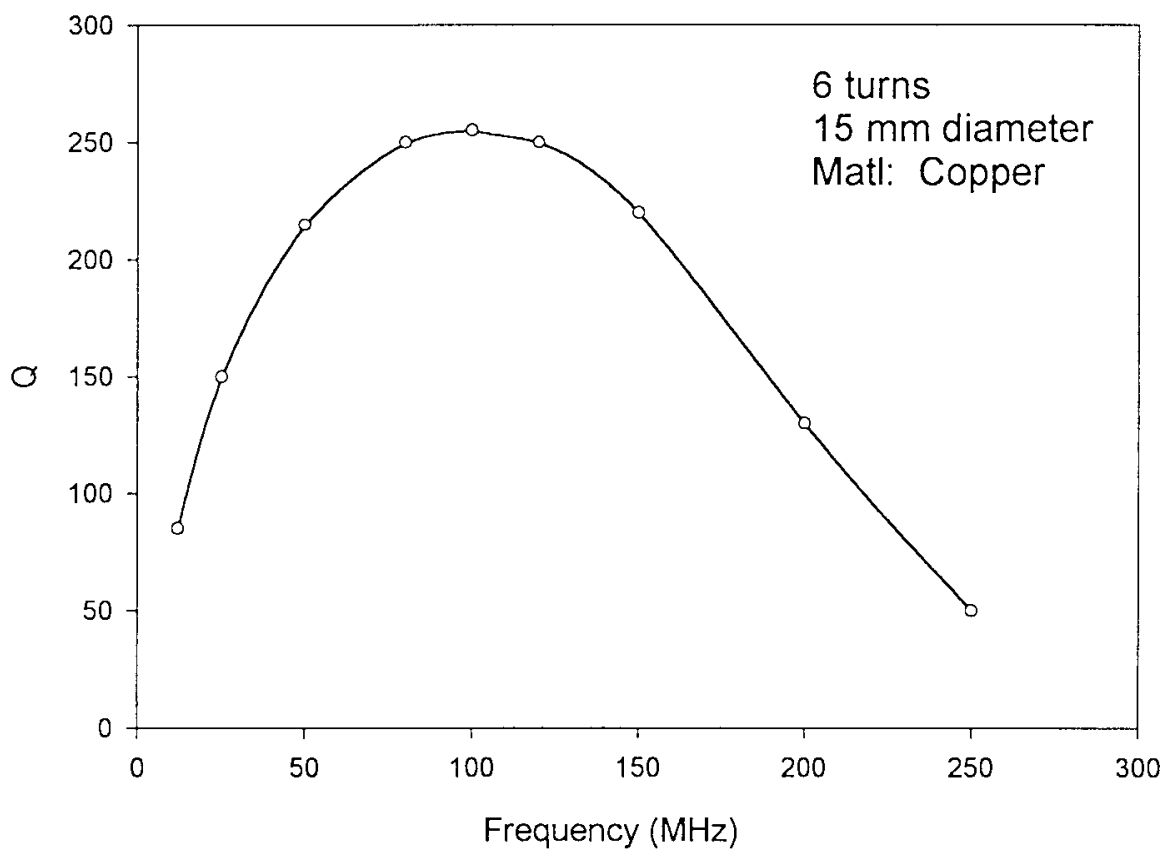

Fig. 1. Calculated effective quality factor $Q(f)$ for a $15 \mathrm{~mm}$ coil (without a plasma load) shows a maximum at $100 \mathrm{MHz}$. The decrease in $Q(f)$ at high frequency is due to self-resonance.

Langmuir probe method [4]. The electron temperature was approximately $10 \mathrm{eV}$ and the ion density $\sim 10^{10} \mathrm{~cm}^{-3}$ using 1 torr Ar and $3 \mathrm{~W}$ of $13.56 \mathrm{MHz}$ power. A considerably more intense discharge could be generated at higher power, but even at $3 \mathrm{~W}$ the helical coil's excessive temperature limited plasma operation to a few minutes. In this configuration much of the power is dissipated in the coil rather than in the plasma. This is a common concern in large-scale ICP's as well [5] since the power deposited in the plasma ( $\left.P_{\text {plasma }}\right)$ is limited by the resistance [6] of the plasma generating circuit $\left(R_{\text {system }}\right)$

$$
P_{\text {plasma }}=\frac{R_{\text {plasma }}}{R_{\text {plasma }}+R_{\text {system }}}\left(P_{f}-P_{r}\right)
$$

where $R_{\text {plasma }}$ is the resistance of the plasma, $P_{f}$ is the forward power to the source, and $P_{r}$ is the power reflected from the source. The efficiency of this preliminary plasma source was poor due to the large parasitic resistance of the 20turn coil and large RF coil currents due to the low operating frequency.

\section{B. Spiral Coil Design}

The key to creating an efficient miniature ICP is controlling the resistance of the coil by designing a proper coil geometry and operating the ICP at the optimum frequency. In addition to improved efficiency, it is also desirable to use a planarspiral coil since this shape is compatible with microfabrication techniques. The approach used to design the spiral coil was to choose a coil diameter and then optimize the number of turns, the gap between the turns, and the operating frequency such that the quality factor is maximized. The quality factor was determined from the ratio of the equivalent series reactance to the equivalent series resistance [7] so that self-resonance effects are included. An approximate expression for the quality factor is

$$
Q \approx \frac{2 \pi f L_{\mathrm{TOT}}}{R_{\mathrm{TOT}}}\left[1-\left(\frac{f}{f_{R}}\right)^{2}\right]
$$

where the resonant frequency $\left(f_{R}\right)$ of the coil is

$$
f_{R}=1 / \sqrt{4 \pi^{2} L_{\mathrm{TOT}} C_{P}}
$$

As mentioned above, the total resistance of the coil should be minimized to achieve the best power transfer efficiency to the plasma. The total series resistance is comprised of three components

$$
R_{\mathrm{TOT}}=R_{\mathrm{DC}}+R_{\mathrm{SE}}(f)+R_{\mathrm{PE}}(f)
$$

which describe the dc resistance of the coil (DC), the skin effect (SE), and the proximity effect (PE) as described in Snelling [8] and Rodriguez et al. [7]. The total inductance ( $L_{\mathrm{TOT}}$ ) of the coil is comprised of internal wire inductance, self inductance of each turn, and mutual inductance between turns. The parasitic capacitance $\left(C_{P}\right)$ between coil turns is approximated from the capacitance between parallel conductors of cylindrical cross section. Both $L_{\mathrm{TOT}}$ and $R_{\mathrm{TOT}}$ are calculated by assuming the coil is constructed from interconnected concentric rings [see (7)].

The theoretical quality factor for a typical $15 \mathrm{~mm}$ diameter spiral is shown in Fig. 1. The general trend is for $Q(f)$ to increase with frequency until self-resonance is approached. For the example shown, the highest quality factor occurs for $f=100 \mathrm{MHz}$. The plasma source built using this coil design operates between $100 \mathrm{MHz}$ and $130 \mathrm{MHz}$. 

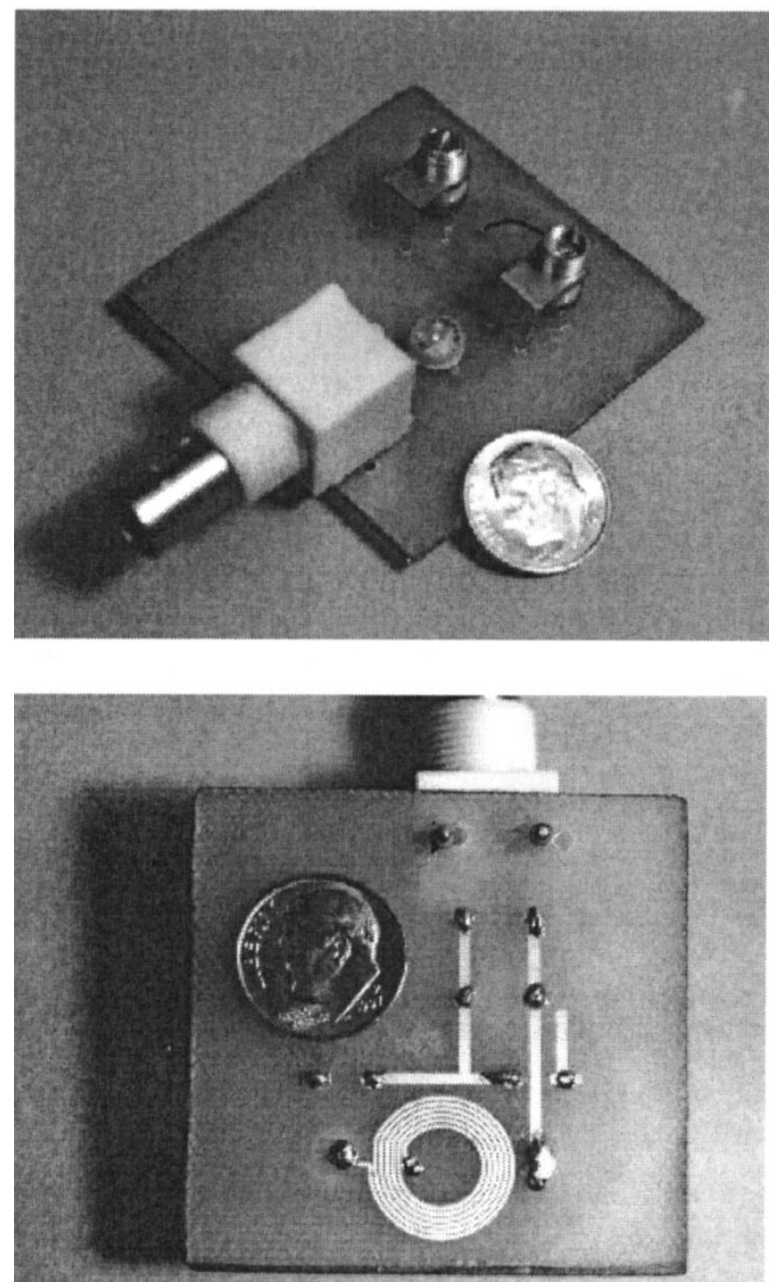

Fig. 2. Top and bottom view of a miniature ICP circuit fabricated on a copper clad epoxy board. A U.S. dime $(\phi=18 \mathrm{~mm})$ is shown as a scale reference.

\section{Miniature Planar ICP Sources}

Spiral inductors were patterned on $1 \mathrm{oz}$ copper clad epoxy boards using photoresist and a contact mask aligner. The copper was spray etched using ferric chloride in water $(1: 3)$. This process resulted in a minimum line gap of approximately $100 \mu \mathrm{m}$ in $\sim 30 \mu \mathrm{m}$ thick copper. Thicker $2 \mathrm{oz}$ copper films were also used to construct the coils, but the minimum gap increased during the longer isotropic copper etch. As a result, the surface area of the coil was unchanged and no difference was observed in the performance of the ICP sources using 1 or $2 \mathrm{oz}$ copper. Two high voltage, high $Q$, variable capacitors provided the impedance matching of the RF power source $(Z=50 \Omega)$ to the plasma source. Fig. 2 shows the top and bottom view of a complete ICP source. In addition to this 15 $\mathrm{mm}$ diameter source, two other coil sizes were investigated as detailed in Table I.

The spiral coils were tested using a small vacuum system consisting of a stainless steel cross as shown in Fig. 3. The coil was positioned adjacent to a $15 \mathrm{~mm}$ diameter window of 7056 glass with a thickness of $1.8 \mathrm{~mm}$. Argon and air were introduced through a needle valve and the gas pressure was measured by a high accuracy capacitance manometer. Plasma power was controlled by a HP 8656A signal generator (1-990
TABLE I

Parameters for the Three Miniature Colls Used in This Work

\begin{tabular}{cccccc}
\hline $\begin{array}{c}\text { Coil diam } \\
(\mathrm{mm})\end{array}$ & $\begin{array}{c}\text { Number of } \\
\text { turns }\end{array}$ & $\begin{array}{c}\text { Inductance } \\
(\mathrm{nH})\end{array}$ & $\begin{array}{c}\text { Width of the } \\
\text { conductor }(\mu \mathrm{m})\end{array}$ & $\begin{array}{c}\text { Gap between } \\
\text { turns }(\mu \mathrm{m})\end{array}$ & $\begin{array}{c}\text { Max } \\
\mathrm{Q}\end{array}$ \\
\hline 5 & 3 & 36 & 200 & 100 & 240 \\
10 & 5 & 200 & 200 & 200 & 290 \\
15 & 6 & 720 & 450 & 140 & 260 \\
\hline
\end{tabular}

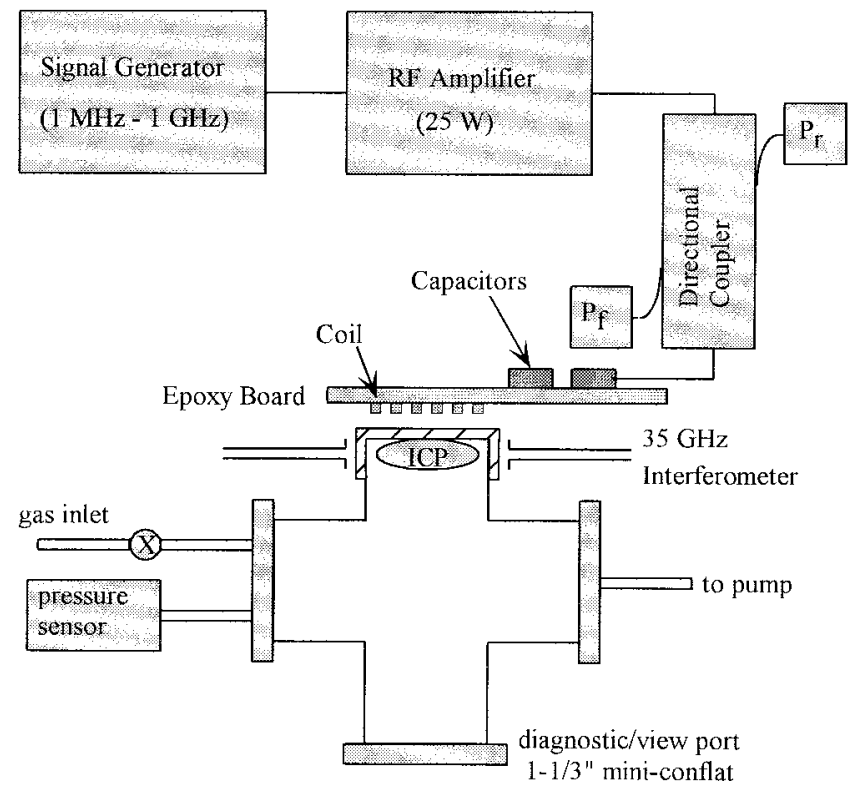

Fig. 3. Schematic of the experimental system used to test miniature ICP's. The plasma diameter is $15 \mathrm{~mm}$.

MHz) and amplified by a ENI 525LA $25 \mathrm{~W}$ linear amplifier. The forward and reverse power were monitored using a Narda 3020A dual directional coupler and a HP 435A power meter.

Optical and Langmuir probe diagnostics were introduced through a $1-1 / 3$ in conflat flange on the Tee opposite the plasma source. The single Langmuir probe was constructed from a tungsten wire $(0.25 \mathrm{~mm} \phi \times 4 \mathrm{~mm})$ that was sealed into the end of a $6 \mathrm{~mm}$ Pyrex tube. A second-order LC filter in series with the probe was tuned to the frequency of the power supply to eliminate RF distortion of the probe's current-voltage characteristic. The electron temperature $\left(T_{e}\right)$ was determined from the slope of the i-v curve in the electron retardation region after subtraction of the ion current. The plasma potential $\left(V_{P}\right)$ was determined from the probe voltage at which $d^{2} i / d v^{2}=0$, and the electron density $\left(n_{e}\right)$ was found from the electron saturation current at $v=V_{P}$ [9]. Probe measurements were not taken above $\sim 0.5$ torr due to violation of the collisionless sheath assumption implicit in the data analysis. A $35 \mathrm{GHz}$ microwave interferometer was used to confirm the Langmuir probe measurements by passing an electromagnetic wave across the plasma's diameter as shown in Fig. 2. The interferometer also allowed the average electron density to be determined at gas pressures above 0.5 torr. 


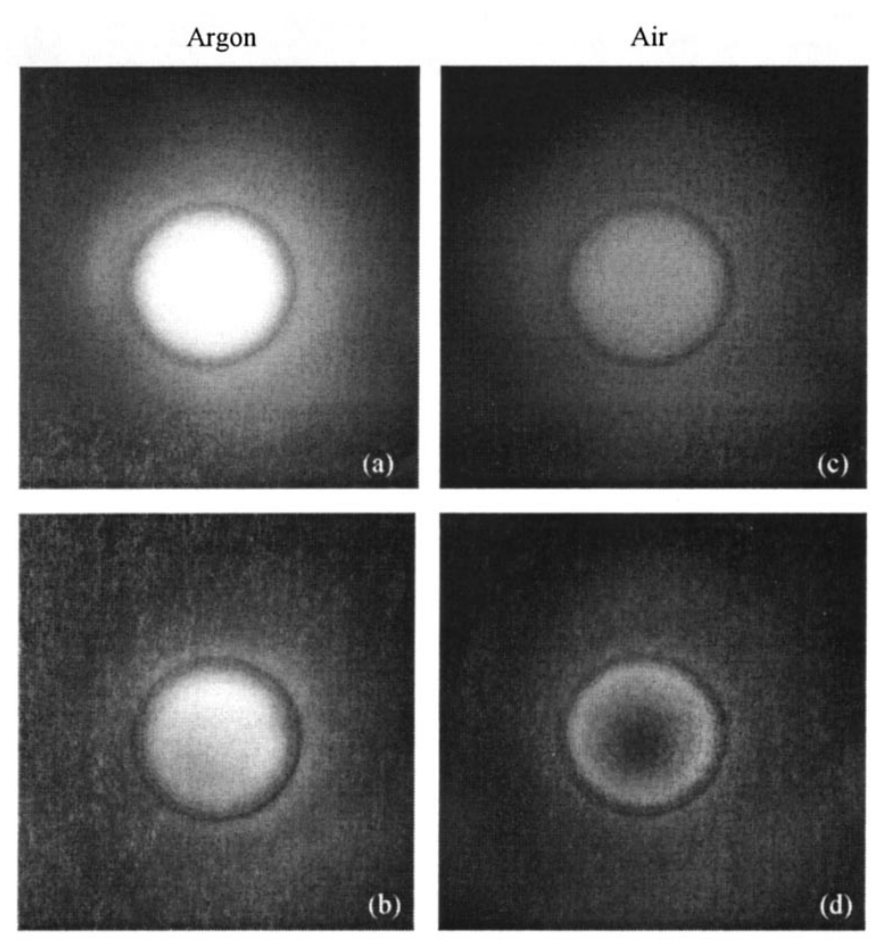

Fig. 4. Photos of argon and air plasmas using $2 \mathrm{~W}$ RF at 0.02 torr (top) and 1.4 torr (bottom). The operating frequency was approximately $120 \mathrm{MHz}$.

\section{RESUltS AND DisCUSSION}

\section{A. Discharge Appearance}

Fig. 4 shows photos of ICP's created by a $15 \mathrm{~mm}$ coil as viewed from the viewport opposite the coil. Photos (a) and (c) were taken at 0.02 torr while (b) and (d) represent 1.4 torr discharges. Both the argon and air discharges appear diffuse and relatively uniform at pressures less than 1 torr. Some interesting structure is visible in the plasma at higher pressures. The air plasma develops a ring-like shape characteristic of early planar inductive plasmas [10]. The ring of plasma indicates that excitation occurs in the ring-shaped electric field region created by the coil [11]. At high pressures the plasma species de-excite before they can diffuse toward the center of the plasma. The ring structure is less evident in the 1.4 torr argon plasma. At 10 torr Ar, however, three small striations are observed to form around the chamber periphery. These striations appear similar to those reported by Stittsworth and Wendt in a $360 \mathrm{~mm}$ plasma at 0.085 torr [12].

\section{B. Plasma Ignition}

In MEMS applications the gas pumping speed is generally quite poor and a plasma source will need to operate at high pressure. Alternatively, in space applications the pumping of gas is not a problem, but because gas utilization is a concern, low pressure operation is important. These two applications point out the need to understand the parameters that effect plasma ignition as a function of pressure.

The relationship between the power supply frequency and the minimum power required to initiate the plasma is shown in Fig. 5. The chamber dimensions were not changed in this study, but the frequency was selected from $130 \mathrm{MHz}, 220$ $\mathrm{MHz}$, or $460 \mathrm{MHz}$ by reducing the coil diameter. At each operating frequency there is a gas pressure at which the plasma is most easily started. This minimum was found to occur when the electron elastic collision frequency is approximately equal to the power supply's radian frequency $(v=\omega=2 \pi f)$. The condition that $v=\omega$ corresponds with the maximum ohmic power absorption from an RF field by an unmagnetized plasma. The elastic collision frequency is computed from $v=K_{\mathrm{el}} n_{g}$, where $n_{g}$ is the argon gas density at room temperature $\left(3.5 \times 10^{22} \mathrm{~m}^{-3}\right.$ per torr $)$ and $K_{\mathrm{el}} \sim 10^{-13} \mathrm{~m}^{3} \mathrm{~s}^{-1}$ is the rate constant for elastic collisions with argon in the range of the measured electron temperature [13]. Arrows on Fig. 5 indicate the pressure at which $v=\omega$ for each operating frequency.

Because any reduction of the coil diameter is accompanied by an increase in operating frequency, the ideal plasma ignition pressure scales up as the coil dimension is reduced. The ideal pressure should be 1-2 torr for MEMS ICP's. This gas pressure is appropriate for gas analysis applications, but will likely be too high for ion beam sources and thrusters, suggesting that further technological innovations are needed. Also note that the $5 \mathrm{~mm}$ coil required a higher total power to start the discharge compared to the larger coils. One reason higher power was needed is that the window thickness was not scaled with the coil diameter in this experiment. Ideally, the window thickness should be much less than the coil radius such that the inductive field strength does not decay too severely before reaching the window-plasma interface (see [5]). Poor field penetration into the plasma region when the coil radius $(2.5 \mathrm{~mm})$ is comparable to the window thickness $(1.8 \mathrm{~mm})$ is a reason that the smallest ICP did not start as easily.

\section{Electron Density}

Langmuir probe measurements of the electron density in a 0.14 torr argon plasma are compared with the average electron density as determined by microwave interferometry [14] in Fig. 6. The probe was located at the center of the discharge along the axis and sampled electrons from the 4 $\mathrm{mm}$ region nearest the coil. This central region is expected to yield the highest electron density when the plasma is diffuse at low pressure. The interferometer measures the line-integrated electron density $\left(\mathrm{m}^{-2}\right)$ across the diameter of the plasma which is then reported as the average electron density $\left(\mathrm{m}^{-3}\right)$ after dividing by the plasma diameter. The error bar represents the uncertainty introduced by the minimum phase resolution of the interferometer. Additional uncertainty exists due to the small size of the plasma in relation to the wavelength emitted by the interferometer.

The plasma density determined by each technique is consistent, but it is approximately an order of magnitude less than that reported for large-scale ICP's at the same pressurelength product and power density [15], [16]. There are three explanations for the low electron density: 1) the surface area-to-volume ratio of the small plasmas is high, resulting in increased electron-ion wall recombination, 2) the power efficiency is low due to resistive power loss in the coil, and 


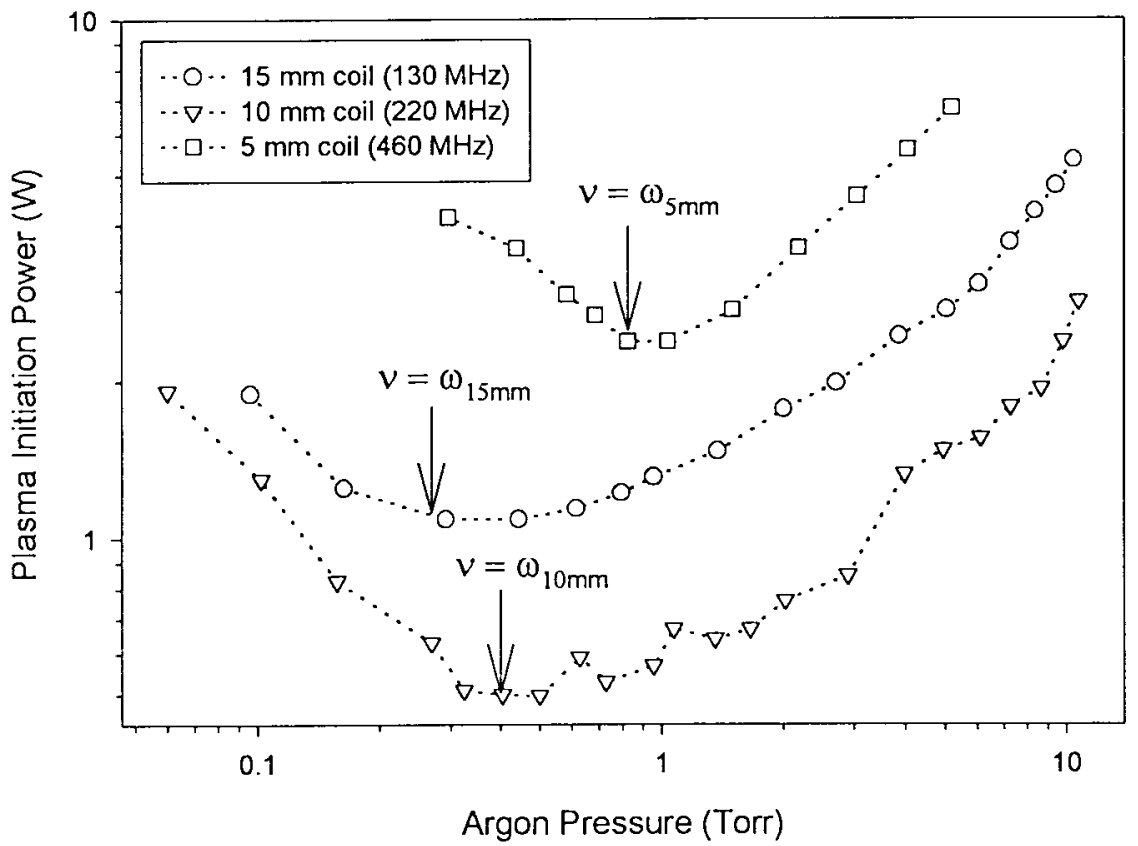

Fig. 5. Power required for plasma ignition shows a distinct minimum versus pressure. The minimum occurs when the electron elastic collision frequency equals the RF source frequency $(v=\omega)$ as shown by the arrows.

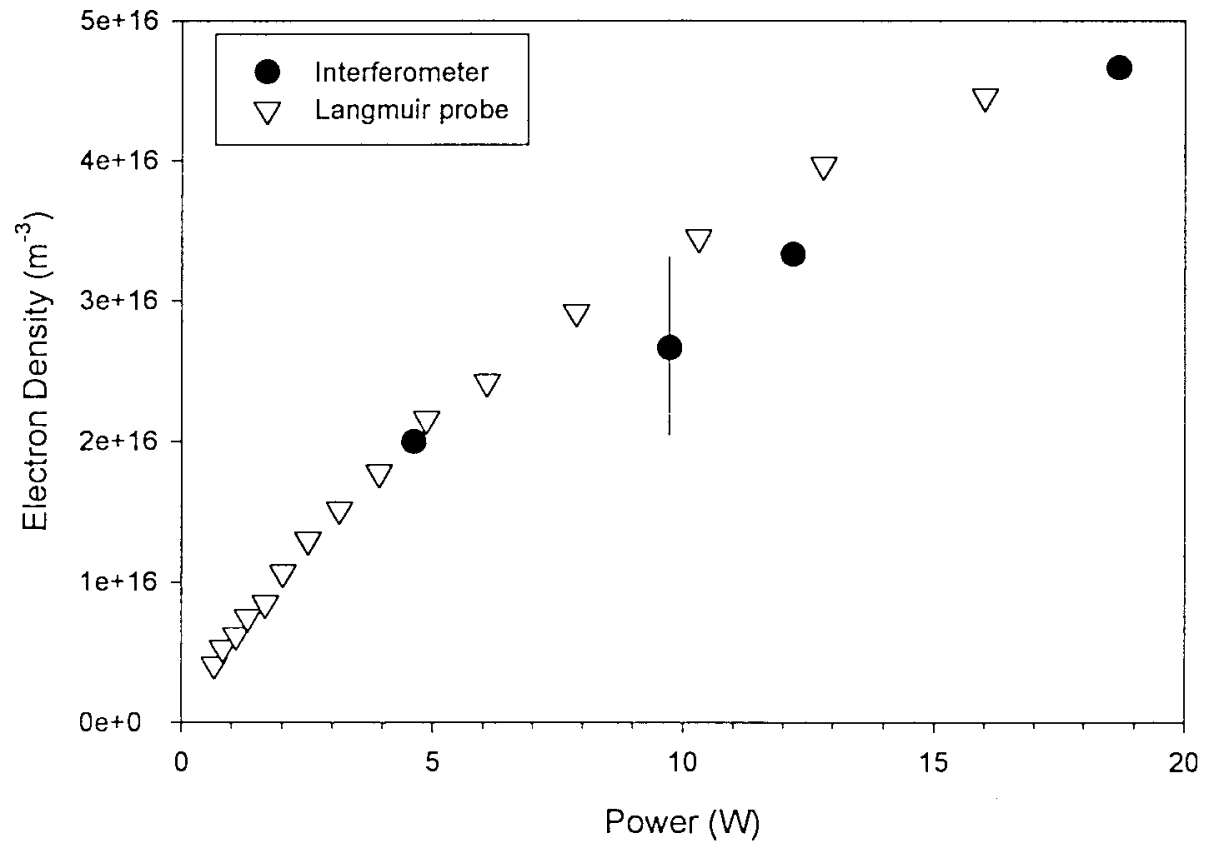

Fig. 6. The electron density determined by Langmuir probe at the center of a 0.14 torr argon discharge compares favorably with the average electron density as measured by microwave interferometry $(15 \mathrm{~mm}$ coil, $f=120 \mathrm{MHz})$.

3) the miniature plasmas may have a strong component of capacitive coupling that wastes RF power by accelerating charged species toward the coil rather than generating electronion pairs.

Although capacitive coupling cannot be conclusively eliminated as the cause of lower-than-expected density, a capacitively coupled discharge will exhibit a strong increase in RF floating potential with RF power as well as some dependence in the plasma potential. Probe measurements, however, show that the plasma potential, the electron temperature, and the RF component of the floating potential are essentially independent of RF power. Power efficiency of the coil is not quantified yet, but most of the power is absorbed by the plasma since the source can be operated continuously at several watts without excessive heating of the coil or the tuning circuit. Finally, the surface-to-volume ratio of the current ICP is $\sim 4.5 \mathrm{~cm}^{-1}$. In contrast, a typical large-scale ICP [17] has a surface-to-volume ratio of only $\sim 0.3 \mathrm{~cm}^{-1}$. This simple calculation shows that 


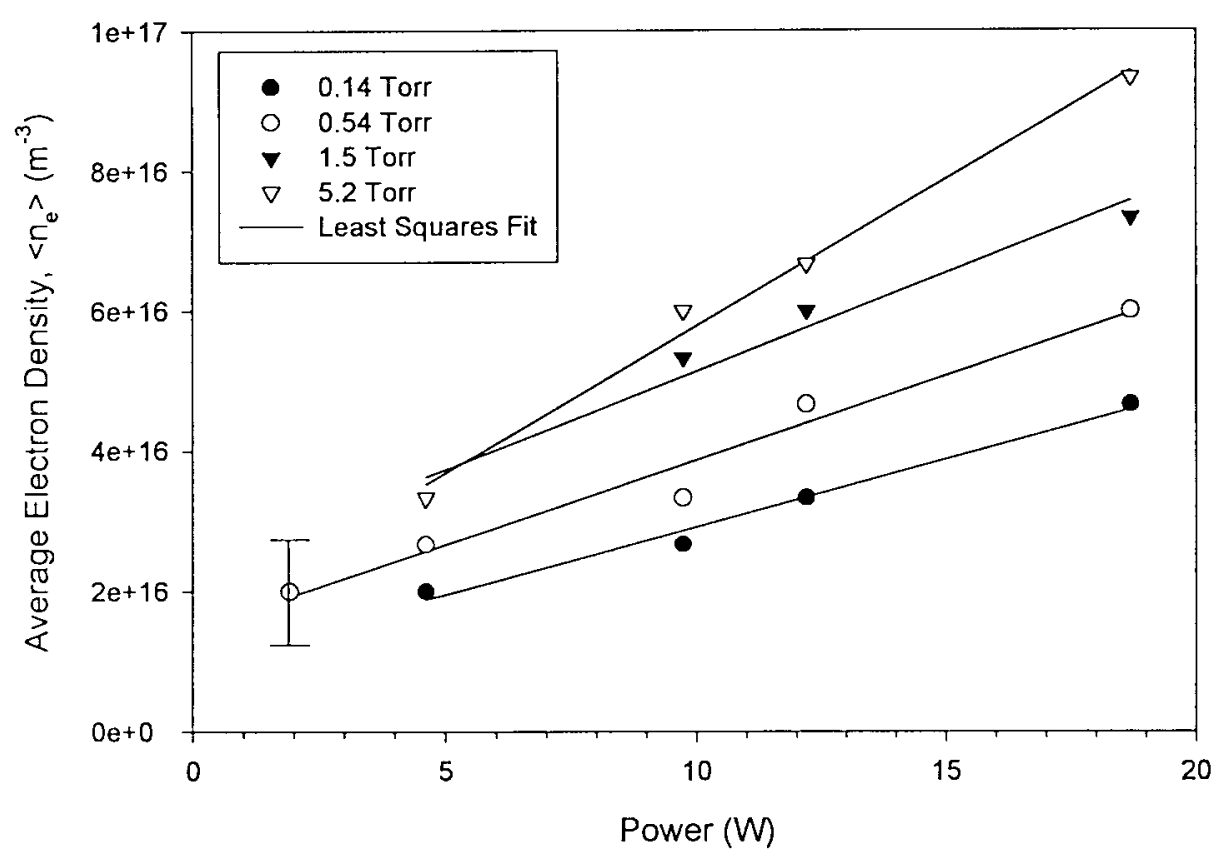

Fig. 7. Microwave interferometry is used to determined the electron density in a miniature argon ICP at high pressure (coil diameter $=15 \mathrm{~mm})$.

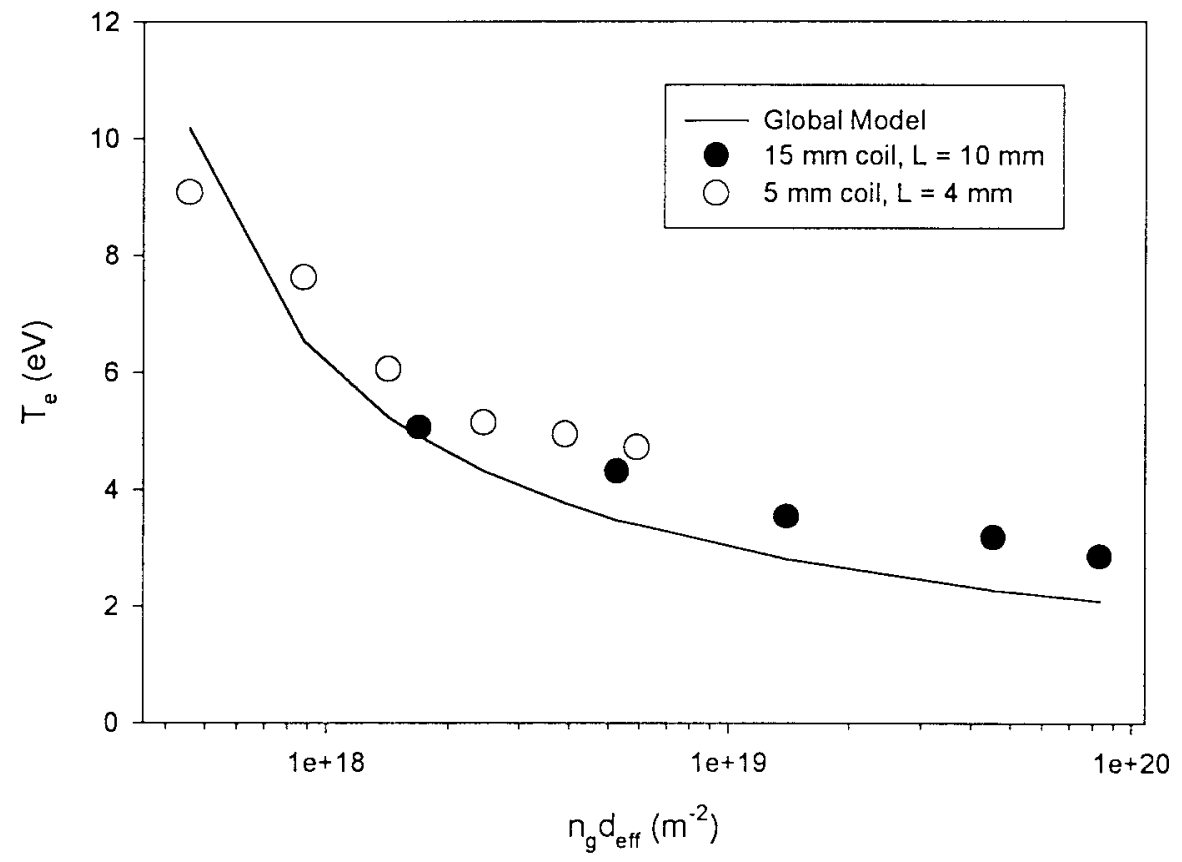

Fig. 8. The measured electron temperature of two miniature ICP's agrees closely with the global model only when the size of the plasma is determined by subtracting the sheath width from the chamber dimensions.

the surface recombination rate per unit plasma volume will be about an order of magnitude greater in the miniature ICP. This accelerated loss rate is believed to cause the order-ofmagnitude decrease in electron density. A more detailed global model [18] based on energy balance verifies this simple scaling argument, although the global model overpredicts the electron density.

The electron density could not be determined at high pressure using the Langmuir probe since the collisional collection of charged particles is difficult to interpret. The average electron density as determined by interferometry, however, is shown in Fig. 7 up to a pressure of 5 torr. Increased pressure results in higher electron density. This is an encouraging observation in the context of high pressure, scaled-down ICP's.

\section{Electron Temperature}

The electron temperature in the miniature ICP is found to vary between $3 \mathrm{eV}$ and $9 \mathrm{eV}$ in argon. The usual, qualitatively observed increase in electron temperature occurs at both lower 


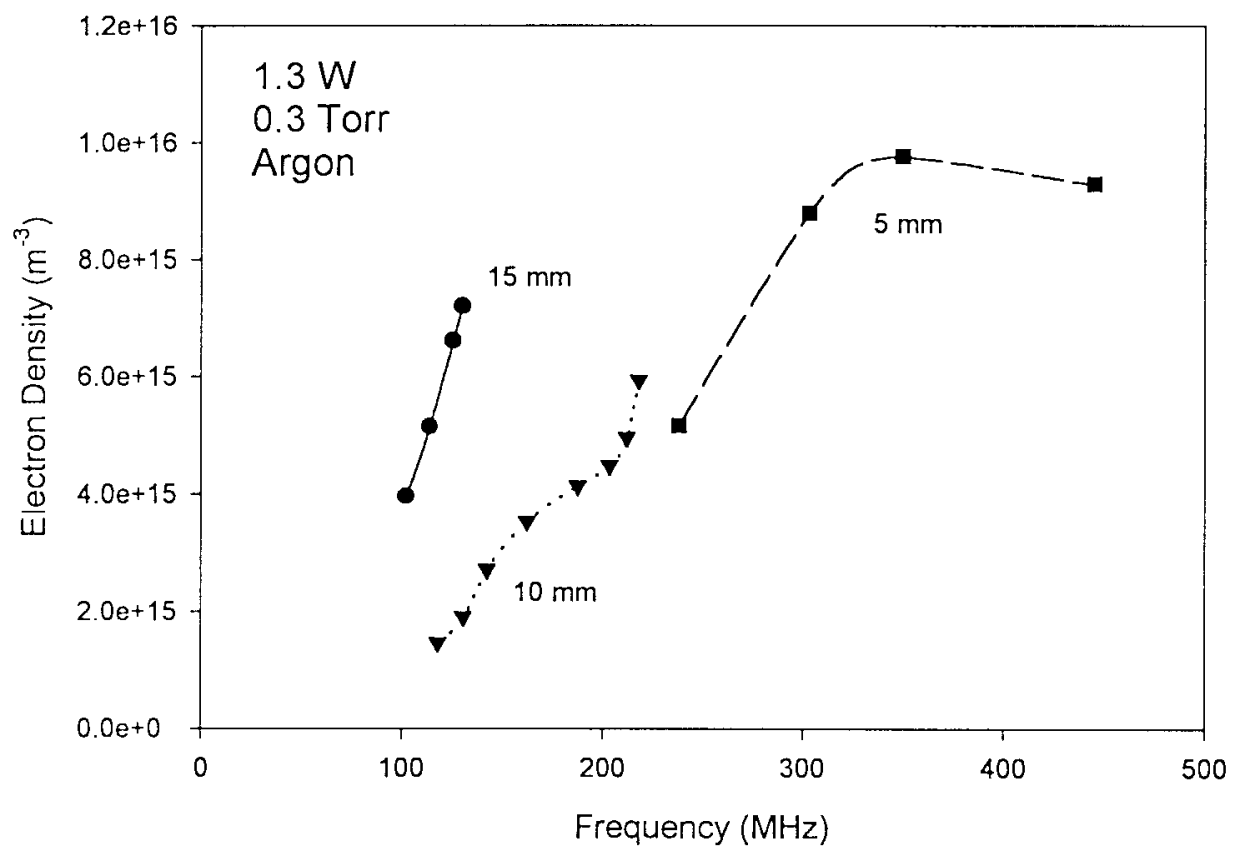

(a)

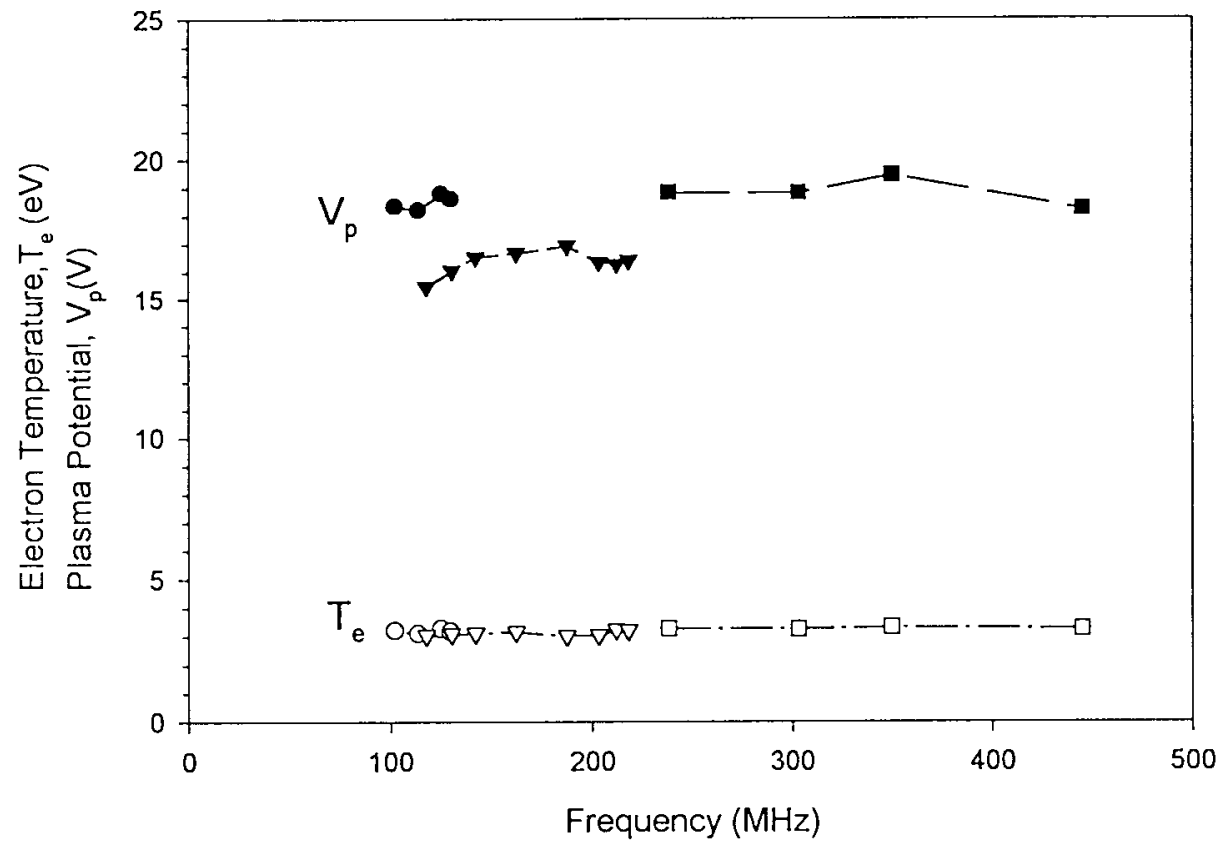

(b)

Fig. 9. (a) The electron density, as determined by Langmuir probe, shows a strong dependency on power supply frequency. (b) Since the electron temperature and plasma potential are independent of frequency, the ionization rate and energy loss rate are not frequency dependent.

pressure and in smaller volume plasmas. To determine more general scaling laws for MEMS-compatible ICP's, the electron temperature data are plotted in Fig. 8 along with the global model [18] for plasmas that is derived from particle balance. The global model states that the electron temperature is a unique function of gas density $\left(n_{g}\right)$ and the effective plasma size $\left(d_{\mathrm{eff}}\right)$

$$
\frac{K_{\mathrm{iz}}}{u_{B}} \approx \frac{5 \times 10^{-14} e^{-15.7 / T_{e}}}{\sqrt{k T_{e} / m_{i}}}=\frac{1}{n_{g} d_{\mathrm{eff}}}
$$

where the ionization rate constant for argon is $K_{\mathrm{iz}}\left(\mathrm{m}^{3} \mathrm{~s}^{-1}\right)$, $u_{B}$ is the Bohm velocity, $k$ is the Boltzmann constant, and $m_{i}$ is the argon ion mass. The effective plasma size has been determined in previous work from the radius $(R)$ and length $(L)$ of the plasma chamber

$$
d_{\mathrm{eff}}=\frac{R L}{2\left(h_{l} R+h_{R} L\right)}
$$

where $h_{l}$ is the pressure-dependent ratio of the ion density at the top sheath edge to the ion density at the center of the 


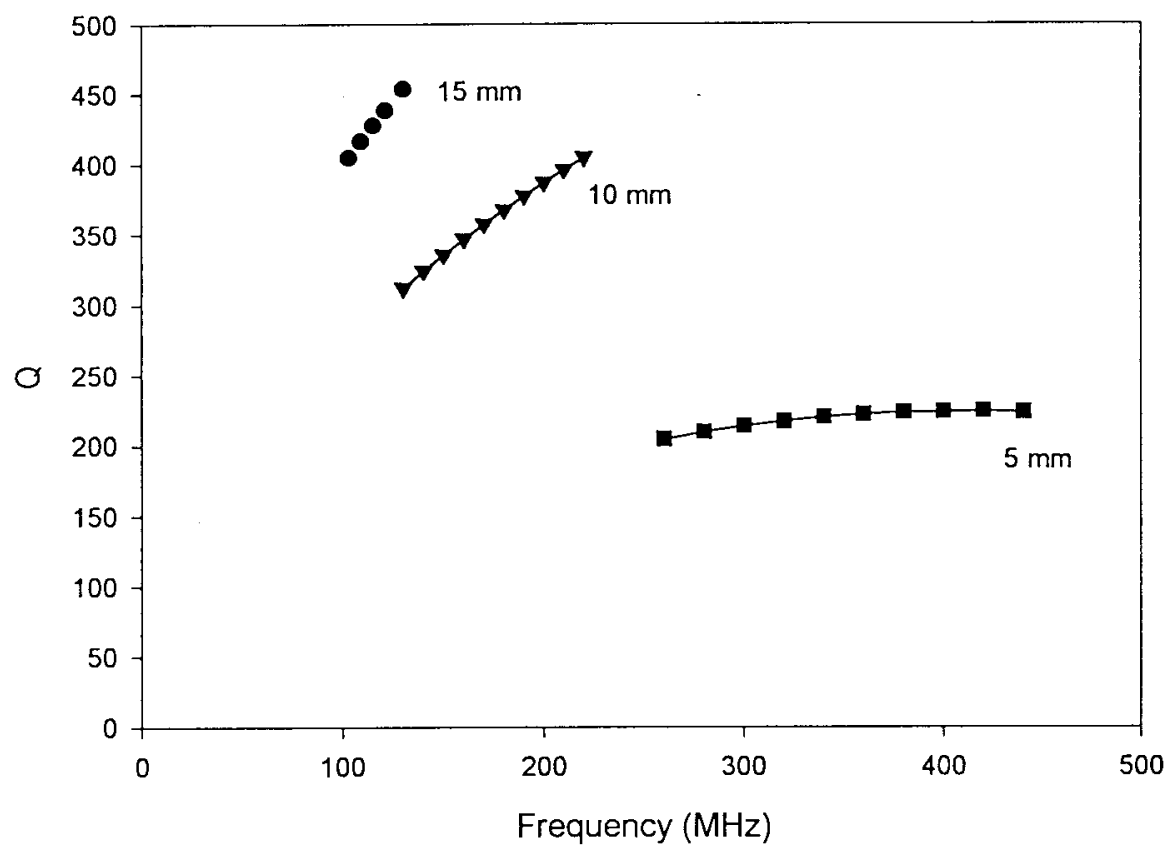

(c)

Fig. 9. (Continued.) (c) The calculated quality factor for each coil shows that the efficiency of power coupling to the plasma improves at higher frequency. (Self-resonance is ignored, $C_{P}=0$.)

plasma, and $h_{R}$ is the ratio of ion density at the radial sheath edge to the center as described by Godyak [19]. The raw data for the miniature plasmas do not follow the model described above unless the plasma sheath length is subtracted from both $R$ and $L$. The plasma sheath width $(s)$ is estimated from the collisionless Child law

$$
s=\frac{\sqrt{2}}{3} \lambda_{D}\left(\frac{2 V_{P}}{T_{e}}\right)^{\frac{3}{4}}
$$

where $\lambda_{D}$ is the electron Debye length and $V_{P}$ is the plasma potential. By making the substitutions $R \leftarrow(R-s)$ and $L \leftarrow$ $(L-2 s)$, the measured electron temperatures are accurately predicted by the global model as shown. The subtraction of the sheath width is generally overlooked in large scale plasmas since the radius and length are much greater than $s$. In small scale discharges of this nature, the plasma dimensions are comparable to the sheath width $(\sim 1 \mathrm{~mm})$. This results in an electron temperature that is somewhat higher than predicted on the basis of the chamber dimensions alone. The important general scaling principle revealed by this data is that the sheath width does not scale with the plasma size. In fact, since the electron density in smaller ICP's decreases, the sheath width becomes greater as the plasma is scaled down. A significant portion of the chamber volume may be occupied by the plasma sheath, resulting in a smaller than expected plasma volume and a higher electron temperature.

\section{E. Frequency Effects}

The excitation frequency of the miniature ICP is continuously varied from $100 \mathrm{MHz}$ to $460 \mathrm{MHz}$ in Fig. 9 by changing the capacitances used in the tuning network and decreasing the diameter (and inductance) of the coil. The upper frequency limit for each coil was reached when the reflected power $\left(P_{r}\right)$ could no longer be nulled by tuning the capacitances. Fig. 9(a) shows the somewhat surprising result that the electron density increases with frequency. The increased electron density is not due to a higher ionization rate since the electron temperature remains constant as shown in Fig. 9(b). The energy loss rate from the plasma per electron-ion pair depends on the plasma potential $\left(V_{P}\right)$. It can also be seen in Fig. 9(b) that $V_{P}$ is approximately constant, and therefore the energy loss rate from the plasma is roughly independent of the frequency. Since generation and loss rates within the plasma are unaffected by frequency, the mechanism responsible for increased electron density must lie outside the plasma. One reason that the electron density improves with frequency is the efficiency of power coupling to the plasma [see (1)]. The quality factor calculated for each coil, as plotted in Fig. 9(c), shows that the system resistance decreases with increasing frequency $\left(Q \sim 1 / R_{\text {system }}\right)$. As the frequency increases for each ICP, more power is available to the plasma as less is dissipated in the coil. Although further study is warranted, it appears from the data in Fig. 9 that the efficiency of the miniaturized ICP is strongly dependent on low-loss coil design and careful selection of the operating frequency.

\section{CONCLUSION}

Planar inductively coupled plasma sources have been scaled-down, fabricated, and characterized. The smallest ICP uses a $5 \mathrm{~mm}$ diameter coil and operates at $460 \mathrm{MHz}$. The discharges have been tested in both argon and air over a pressure range from 0.01 torr to 10 torr. The plasmas can be initiated and sustained with as little as $0.5 \mathrm{~W}$, but also operate as high as $20 \mathrm{~W}$. 
The primary scaling issues reported in this work are 1) the plasma starts most readily when $v=\omega, 2$ ) the plasma sheath width does not scale-down, limiting the ultimate chamber dimension to be larger than the sheath width, 3) the large surface-to-volume ratio of small ICP's decreases the electron density, and 4) the plasma density is considerably more sensitive to the resistance of the coil circuit than large-scale ICP's.

Future work will focus on continued scaling of the coil diameter and reduction in the physical dimensions of the plasma chamber. To accomplish these goals, the source and chamber will be fabricated monolithically using MEMS fabrication techniques.

\section{ACKNOWLEDGMENT}

The authors wish to thank M. Miller, R. Morrison, and W. $\mathrm{Hu}$ for their valuable assistance.

\section{REFERENCES}

[1] R. M. Caloi and C. Carretti, "Getters and gettering in plasma display panels," J. Vac. Sci. Technol. A, vol. 16, pp. 1991-1996, 1998.

[2] B. S. Ross, D. M. Chambers, G. H. Vickers, P. Yang, and G. M. Hieftje, "Characterization of a 9-mm torch for inductively coupled plasma mass spectrometry," J. Anal. At. Spectrom., vol. 5, pp. 351-358, 1990.

[3] J. Mueller, J. R. Brophy, J. E. Polk, and J. J. Blandino, "The JPL ion thruster-on-a-chip concept," presented at the 7th NASA-OSAT Advanced Space Propulsion Workshop, Pasadena, CA, Apr. 10, 1996.

[4] M. A. Lieberman and A. J. Lichtenberg, Principals of Plasma Discharges and Materials Processing. New York: Wiley, 1994, p. 181.

[5] J. Hopwood, "Planar if induction plasma coupling efficiency," Plasma Sources Sci. Technol., vol. 3, pp. 460-464, 1994.

[6] R. B. Piejak, V. A. Godyak, and B. M. Alexandrovich, "A simple analysis of an inductive RF discharge," Plasma Sources Sci. Technol., vol. 1, pp. 179-186, 1992

[7] R. Rodriguez, J. M. Dishman, F. T. Dickens, and E. W. Whelan, "Modeling of two-dimensional spiral inductors," IEEE Trans. Comp., Hybrids, Manufact. Technol., vol. CHMT-3, pp. 535-541, 1980.

[8] E. C. Snelling, Soft Ferrites. London, U.K.: Iliffe, 1969.

[9] B. Chapman, Glow Discharge Processes. New York: Wiley, 1980, pp. $60-70$.

[10] M. McKinnon, "On the origin of the electrodeless discharge," Phil. Mag., vol. 8, pp. 605-616, 1929.

[11] J. Hopwood, C. R. Guarnieri, S. J. Whitehair, and J. J. Cuomo, "Electromagnetic fields in an rf induction plasma," J. Vac. Sci. Technol. A, vol. 11, no. 1, pp. 147-151, 1993.

[12] J. A. Stittsworth and A. E. Wendt, "Striations in a radio frequency planar inductively coupled plasma," IEEE Trans. Plasma Sci., vol. 24, pp. 125-126, 1996

[13] M. A. Lieberman and A. J. Lichtenberg, Principals of Plasma Discharges and Materials Processing. New York: Wiley, 1994, p. 80

[14] D. I. C. Pearson, G. A. Campbell, C. W. Domier, and P. C. Efthimion, "A microwave interferometer for density measurement and stabilization in process plasmas," Mat. Res. Soc. Symp. Proc., 1988, vol. 117, pp. 311-317.
[15] J. Hopwood, C. R. Guarnieri, S. J. Whitehair, and J. J. Cuomo, "Langmuir probe measurements in an rf induction plasma," J. Vac. Sci. Technol. A, vol. 11, pp. 152-156, 1993

[16] L. J. Mahoney, A. E. Wendt, E. Barrios, C. J. Richards, and J. L. Shohet, "Electron-density and energy distributions in a planar inductively coupled discharge," J. Appl. Phys., vol. 76, pp. 2041-2047, 1994.

[17] N. Forgotson, V. Khemka, and J. Hopwood, "Inductively coupled plasma for polymer etching of $200 \mathrm{~mm}$ wafers," J. Vac. Sci. Technol. B, vol. 14, pp. 732-737, 1996

[18] M. A. Lieberman and A. J. Lichtenberg, Principals of Plasma Discharges and Materials Processing. New York: Wiley, 1994, pp. 306-309.

[19] V. A. Godyak, Soviet Radio Frequency Discharge Research. Falls Church, VA: Delphic, 1986, p. 99.

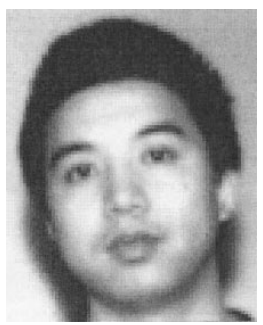

Yu Yin received the B.S. and M.S. degrees in physics in 1992 and 1995, respectively, from Fudan University at Shanghai. Currently, he is working toward the Ph.D. degree in electrical engineering at Northeastern University, Boston, MA.

His interests include inductively coupled plasmas and microfabrication processes.

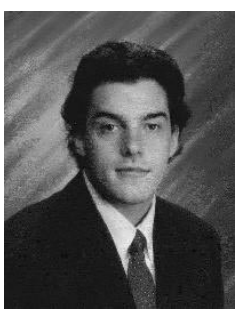

Jason Messier received the B.S. degree in electrical engineering from Northeastern University, Boston, MA, in 1997 and the M.S. degree in electrical engineering from Carnegie Mellon University, Pittsburgh, PA, in 1999.

He is currently working for the Teradyne Corporation in Boston. His interests include computational electromagnetics and mobil robots.

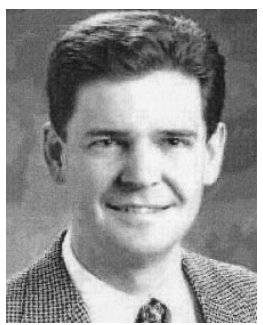

Jeffrey A. Hopwood (S'86-M'90) received the $\mathrm{Ph} . \mathrm{D}$. degree in electrical engineering in 1990 from Michigan State University, East Lansing.

He worked for the IBM Corporation at the T. J. Watson Research Center until 1993 and then joined the faculty at Northeastern University, Boston, MA, where he is currently Associate Professor of Electrical and Computer Engineering. In addition to the study of inductively coupled plasmas, he is interested in ionized physical vapor deposition of high aspect ratio microelectronic interconnects, micromachining of optical materials, and fabrication of three-dimensional integrated circuits. 